\title{
VIII.
}

\section{LIST OF PARTICIPANTS}




\section{LIST OF PARTICIPANTS}

\author{
Y. ACKER \\ INAG \\ 77, Av. Denfert-Rochereau \\ F-75014 Paris \\ France
}

\section{AIME}

Université de Nice

Département d'Astrophysique

Parc Valroge

F-06034 NICE Cedex

France

R. ALBRECHT

ST-ECF

European Southern Observatory

Karl-Schwarzschild-Str. 2

D-8046 Garching bei Mijnchen

B.R.D.

M. ANDEREG

ESTEC

Postbus 299

Ni-2200 AG Noordwijk

The Netherlands

P. ANGEBAULT

European Southern Obgervatory

Karl-Schwarzschild-Str. 2

D-8046 Garching bei München

B.R.D.

J.R.P. ANGEL

University of Arizona

Stevard Observatory

Tucson, AZ 85721

U.S.A.

I. APPENZELLER

Landess ternwarte, Königstuh]

D-6900 Heidelbers 1

$\mathrm{B} \cdot \mathrm{R} \cdot \mathrm{D}$.

A.I. ARDEBERG

Lund Observetory

P.0. Box 1107

S-22104 LUND

Sweden

M. AZZOPARDI

European Southern Observatory

Kar1-Schwarzschild-Str. 2

D-8046 Gerching bei München

B.R.D.

D. BAADE

European Southern Observatory

Karl-Schwarzschild-Str. 2

D-8046 Garching bei München

B.R.D.

K. BAHNER

Max-Planck-Institut fïr Astronomie

Königstuh1

D-6900 Heidelberg

B.R.D.

G. BALIIO

Politecnico di Milano

Dip. Ingegneria Strutturale

Piazza Leonardo da Vinci 32

I-20133 Milano

Italy

R. BANDIERA

European Southern Observatory

Karl-Schwarzschild-Str. 2

D-8046 Garching bei München

B.R.D.
C. BARBIERI

Università di Padova

Istituto di Astronomis

Vicolo dell Osservatorio 5

I-35100 Padova

Italy

L. BARR

Kitt Peak National Observatory

P.0. Box 26732

Tucson, AZ 85726

U.S.A.

H. BARWIG

Universitäts-Sternwarte München

Scheinerstrasse

8000 Minchen 80

B.R.D.

I. BAUDET

European Southern Observatory (ESO)

Casilla 19001

Santiago 19

Chile

J. BECKERS

National Optical Astronomy

Observatories

950 North Cherry Avenue

Tucson, AZ 85726

U.S.A.

S. BECKWITH

Cornell University

Department of Astronomy

Space Science Building

Ithaca, NY 1453-0352

U.S.A.

A. BEHR

Universitäts-Sternwarte

Geismar Landstrasse 11

3400 Göttingen

B.R.D.

S. BENSAMMAR

Observatoire de Meudon

F-92190 Meudon

France

P. BENVENUTI

ST-ECF

European Southern Observatory

Karl-Schwarzschild-Str.

D-8046 Garching bei München

B.R.D.

F. BERTOLA

Osservatorio Astronomico

Vicolo dell 'Osservatorio 5

I-35100 PADOVA

Italy

P. BIEREICHEI

European Southern Observatory

Karl-Schwarzschild-Str. 2

D-8046 Garching bei München

B.R.D.

\section{BINETTE}

European Southern Observatory

Karl-Schwarzschild-Str.

D-8046 Garching bei München

B.R. D.

\section{R.G. BINGHAM}

Royal Greenwich Observatory

Herstmonceux Castl

Hailsham

Eest Sussex BN27 1RP

U. K.
A. BOKSENBERG

Royal Greenwich Observatory

Herstmonceux Castle

Hailsham

East Sussex BN27 1RP

U.K.

E.F. BORRA

Physics Department

Laval University

Quebec, $P Q$

CANADA G1K 7 P4

J.D. BREGMAN

Radiosterrenwacht

Postbus 2

VI-7990 AA Dwingeloo

The Netherlands

J. BREYSACHER

European Southern Observatory

Karl-Schwarzschild-Str. 2

D-8046 Garching bei München

B.R.D.

G. BURBIDGE

Kitt Peak National Observatory

950 North Cherry Avenue

P.0. Box 26732

Tucson, AZ 85726

U.S.A.

H. BUTCHER

Kapteyn Lab.

Postbus 800

NL-9700 AV Groningen

The Netherlands

G. CARPENTIER

INAG Mesures

4, Ave de Neptune

F-95107 Saint-Maur-des-Fosses

France

V. CASTELLANI

Istituto Astronomico

Università "La Sapienza"

Via Lancisi 29

I-00161 Roma

Italy

P. CHARVIN

Observatoire de Paris

61, Av. de 1 'Observatoire

F-75014 Peris

France

A. CHELLI

Observatoire de Lyon

F-69230 Saint-Gents-Laval

France

G. CHINCARINI

European Southern Observatory

Karl-Schwarzschild-Str. 2

D-8046 Garching bei Muinchen

B.R.D.

0. CITTERIO

Istituto Fisica Cosmica/CNR

Via Bassini, 15

I-20133 Milano

Italy

B.C. COSMOVICI

Arbeitsgruppe für Weltraumforschung DFVLR-NE

D-8032 Wessling

B.R.D.

Proceedings of the IAU Colloquium No. 79: "Very Large Telescopes, their Instrumentation and Programs", Garching, April 9-12, 1984. 


\section{G. COUPINOT}

Observatoires du Pic-du-Midi

et de Toulouse

F-65200 Bagnères-de-Bigorre PRANCE

\section{G. COURTES}

Laboratoire d'Astronomie Spatiale du CNRS

Traverse du Siphon

F-13012 Marseille

France

\section{P. CRANE}

Buropean Southern Observatory

Karl-Schwarzschild-Str. 2

D-8046 Garching bei Winchen

B.R.D.

\section{S. CRISTIANI}

European Southern Observatory

Karl-Schwarzsch1ld-Str. 2

D-8046 Garching bei Winchen

B.R.D.

\section{CULLUM}

European Southern Observatory

Karl-Schwarzsch1ld-Str. 2

D-8046 Garching bei Muinchen

B.R.D.

\section{S. D'ODORICO}

European Southern Observatory

Karl-Schwarzschild-Str. 2

D-8046 Garching bei Mïnchen

B.R.D.

A. DANKS

European Southern Observatory (ESO)

Casilla 19001

Santiago 19

Chile

\section{J.I. DANZIGER}

European Southern Observatory

Karl-Schwarzachild-Str. 2

D-8046 Garching bei München

B.R.D.

\section{DE JONGE}

IRAM

Vole 10, Domaine Univeraitaire

F-38406 St. Martin d'Hères

France

\section{H. DEKKER}

Buropean Southern Observatory

Karl-Schwarzachild-Str. 2

D-8046 Garching bei Miinchen

B.R.D.

\section{J. DELHAYE}

Observatoire de Pari

61 Ave de 1 Observatoire

F-75014 Paris

France

\section{M. -H. DEMOULIN-ULRICH}

European Southern Observatory

Karl-Schwarzschild-Str. 2

D-8046 Garching bei Minchen

B.R.D.

\section{DENNEFELD}

Institut d'Astrophysique

98bis Boulevard Arago

F-75014 Parla

France

\section{F. DIEGO}

University College Iondon Dept. of Physics \& Astronomy

Gower Street

London WC1E 6BT

U.K.

\section{A. VAN DIJSSELDONK}

European Southern Observatory

Karl-Schwarzachild-Str. 2

D-8046 Garching bei München B.R.D.

\section{DRAVINS \\ Lund Observatory \\ Box 1107 \\ S-22104 Lund \\ SWEDEN}

\section{DYCK}

Max-Planck-Institut fír Astronomie

Königstuh 1

D-6900 Heidelberg

B.R.D.

\section{BATON \\ ESTEC}

Postbus 299

NL-2200 AG Noordwijk

The Netherlands

D. ENARD

European Southern Observatory

Karl-Schwarzschild-Str. 2

D-8046 Garching bei Minchen

B. R. D.

0. ENGVOLD

University of 0 sio

Institute of Theoretical Astrophysics

P.0. Box 1029

Blindern, OSLO 3

Norway

\section{N. EPCHTEIN}

Observatoire de Meudon

DESPA

F-92195 Meudon Principal Cedex

France

\section{H.W. EPPS}

University of California

Dept. of Astronomy

Los Angeles, California 90024

U.S.A.

\section{I.N. EVANS}

Mount Stromlo and Siding Spring Observatories

Private Beg

Woden P.O., A.C.T. 2606

Australia

G. PINGER

European Southern Observatory

Karl-Schwarzschild-Str. 2

D-3046 Garching bei Minchen

B.R.D.

c. FLEBUS

European Southern Observatory

Karl-Schwarzschild-Str. 2

D-8046 Garching bei Minchen

B.R.D.

\section{A.F. FOMENKO}

Speciel Astrophysical Observatory

Nizhnij Arkhyz

Stavropolskij Kraj 357140

U.S.S.R.

F.F. FORBES

Kitt Peak National Observatory

$950 \mathrm{~N}$. Cherry Ave.

Tucson, AZ 85726

U.S.A.

\section{B.P. FORT}

Observatoire de Toulouse

14, Avenue Edouard Belin

F-31400 Toulouse

France
F. FRANZA

European Southern Observatory

Karl-Schwarzachild-Str. 2

D-8046 Garching bei München

B.R.D.

R. FREIRE FERRERO

Observatoire Astronomique

11. Fue de I'Université

F-67000 Strasbourg

France

K. FRICKE

Universitäts-Sternwarte

Geismarlandstrasse 11

D-3400 Göttingen

B.R.D.

F. FUSI-PECCI

Università di Bologns

Department of Astronomy

Via Zamboni 33

Casella Postale 596

I-40100 Bologna

Italy

W. GALLIENI

ADS Italia

Via Caio Plinio 2

I-22030 LIPOMO (Como)

Italy

E.H. GEYER

Observatorium Hoher Lis

D-5568 Daun

B.R.D.

R. GEYL

R.E.O.S.C.

10 Rue des Ecoles

F-91160 Bellainvilliers

France

D. GILLET

European Southern Observatory Karl-Schwarzschild-Str. 2 D-8046 Garching bei Minchen

B.R.D.

P. GILLINGHAM

Anglo-Australian Observatory

Coonabarabran, NSW 2357

Australie

P. GIORDANO

European Southern Observatory

Kar1-Schwarzschild-Str. 2

D-8046 Garching bei München

B.R.D.

P. GROSB $\varnothing L$

European Southern Observatory Karl-Schwarzschild-Str. 2 D-8046 Garching bei Miinchen B.R.D.

W.A. GRUNDMANN

Dominton Astrophysical Observatory 5071 W. Saanich Road

Victoria, B.C.

CANADA V8X 4M6

\section{B. GUSTAFSSON}

European Southern Observatory

Karl-Schwarzschild-Str. 2

D-8046 Garching bei Minchen

B. R. D.

\section{GUTCKE}

JENOPTIK JENA GmbH

Astro-Applikation

Carl-Zeiss-Strasse 1

DDR-6900 Jena

D.D.R. 
D.N. HALL

University of Hawail

Institute of Astronomy

2680 Woodlawn Drive

Honolulu, HI 96822

U.S.A.

\section{J. HECQUET}

Observatoires du Pic-du-Midi

et de Toulouse

F-65200 Bagnères-de-Bigorre

FRANCE

E. HEYNACHER

CAKL ZEISS

Postfach $1369 / 1380$

D-7082 Oberkochen

B.R.D.

M.C.E. HUBER

Institut fír Astronomie

ETH-Zen trum

CH-8092 Zlirich

Schweiz

C.M. HIMPHRIES

Royal Observatory

Blackford Hill

Edinburgh EH9 $3 \mathrm{HJ}$

Scotland, U.K.

S. ISOBE

Tokyo As tronomical Observatory

2-21-1, 0sawa, Mi taka

TOKYO 181

Japan

J. JOUBERT

INAG

77, Av. Denfert-Rochereau

F-75014 Paris

France

H.J. KARCHER

M.A.N.

Abt. VOA

D-6095 G1nsheim-Gustavsburg

B.R.D.

H. KIMURA

MPI fitr Aeronomie

D-3411 Katlenburg-Lindau

B.R.D.

E. $-D$. KNOHI

Carl Zelss

Postfach 1369

7082 Oberkochen

B.R.D.

W. KOLLATSCHNY

Uni-Sternwarte Göttingen

Geismarlandstr. 1

D-3400 Göttingen

B.R.D.

B.Z. KOVACHEV

Dept. of Astronomy and

National Astronomical Observatory

72 Lenin Blvd.

BG-1184 Sofia

Bulgaria

J. KRAUTTER

Kax-Planck-Institut für extraterrestrische Physik

Kar1-Schwarzschild-Str. 1

D-8046 Garching bei Muinchen

B.R.D.

C. KUHNE

Carl Zeiss

Postfach 1369/1380

7082 Oberkochen

B.R.D.
A. LABEYRIE

CERGA

F-06460 St. Vallier

France

F. JACOMBE

LAIR Observatoire de Meudon

F-92195 Meudon

France

\section{LAURENT}

Institut National d'Astronomie

et de Géophysique - INAG

77 , avenue Denfert-Rochereau

F-75014 Paris

France

\section{S. LAUSTSEN}

Institute of Astronomy

Aarhus University

DK-8000 Aarhus C

Denmark

J. LE GALLO

INAG

77, Av. Denfert-Rochereau

F-75014 Paris

France

R.S. LE POOLE

Sterrewacht Leiden

Huygens Laboratorium

Postbus 9513

NL-2300 RA Leiden

The Netherlands

P. LENA

Observatoire de Paria

F-92190 Neudon

France

J. LEQUEUX

Observatoire de Marseille

2, place Le Verrier

F-13248 Marseilie Cedex 4

France

P. O. LINDBLAD

Stockholms Observatorium

S-13300 Seltsjöbeden

Sweden

C.P. LIU

Max-Planck Institut fïr Aeronomie

D-3411 Katlenburg-Iindau

B.R.D.

J.-I. IIZON A L'ALIEMAND

European Southern Observatory

Karl-Schwarzachild-Str. 2

D-8046 Garching bei Minchen

B.R.D.

\section{S.P. LOPRIORE}

European Southern Observatory

Kar1-Schwarzschild-Str. 2

D-8046 Garching bei Müchen

B.R.D.

\section{LORENZETTI}

Istituto Fisica Spazio

Interplanetario - CNR

I-00044 Frascati

Italy

B. MACK

Royal Greenwich Observatory

Heratmonceux Castle

Hailsham

East Susgex BN27 1RP

U.K.

B. MALECEK

Observatory

CS-757 O1 VALASSKE MEZIRICI

Crechoslovakio

J. MANOUSOYANNAKI

European Southern Observatory

Karl-Schwarzschild-Str. 2

D-8046 Garching bel Mïnchen

B.R.D.

B. MARANO

Università di Bologna

Department of Astronomy

Via Zamboni 33

Casella Postale 596

I-40100 Bologna

Italy

P. MARGUTTI

European Southern Observatory

Kar1-Schwarzschild-Str. 2

D-8046 Garching bet Winchen

B.R.D.

\section{J. -M. MARIOTTI}

Observatoire de Lyon

F-69230 Saint-Gents Laval

France

\section{F. MATTEUCCI}

European Southern Observatory

Karl-Schwarzschild-Str. 2

D-8046 Garching bei Minchen

B.R.D.

E. MAURICE

Observatoire de Marseille

2 , place Le Verrier

F-13248 Marsellle Cedex 4

France

S. MAZZARIOL

European Southern Observatory

Karl-Schwarzschild-Str. 2

D-8046 Garching bei Minchen

B.R.D.

C.R. MCCREIGHT

NASA-Ames Research Center

Mail Stop 244-7

Moffett Field, CA 94035

U.S.A.

C.E. McILWAIN

University of California San Diego

UCSD/CASS/C-011

La Jolla, California 92093

U.S.A.

H. -J. MEIER

Carl Zeiss

Postfach 1369

7082 Oberkochen

B.R.D.

A.B. MEINEL

Optical Sciences Center

O. VON DER LUHE

Univergity of Ar

Kiepenheuer-Institut für Sonnenphysik Tucson, AZ 8572

Schöneckstrasse 6

F-7800 Freiburg/Brsg.

B.R.D.

U.S.A.

F. WIDDELBURG

European Southern Observatory

Kar1-Schwarzsch1ld-Str. 2

D-8046 Garching be1 Minchen

B.R.D. 


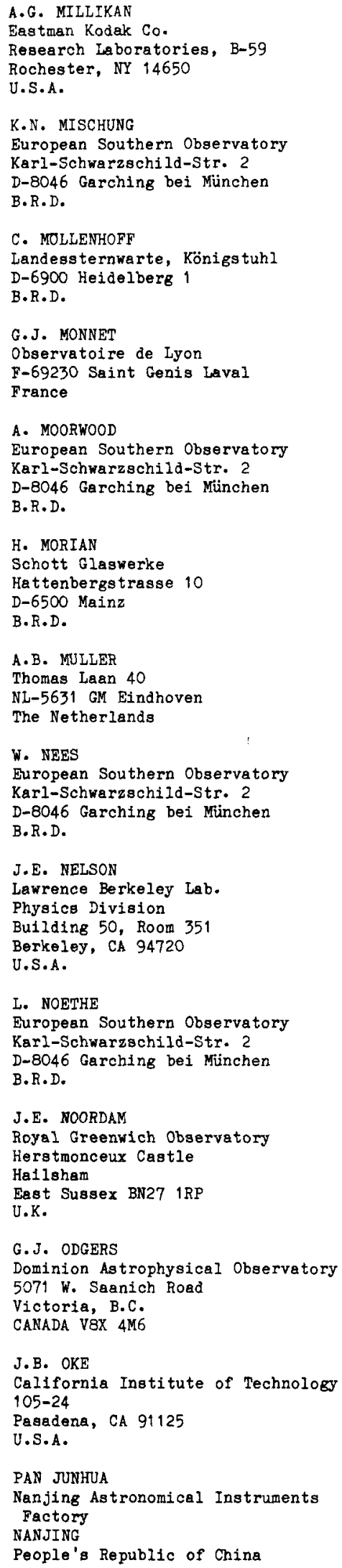

J. PAUREAU

European Southern Observatory

Karl-Schwarzschild-Str. 2

D-8046 Garching bei München

B.P.D.

\section{R.G. PETROV}

Université de Nice

Département d'Astrophysique

Parc Valrose

F-06034 NICE Cedex

France

D. PLATHNER

IRAI

Voie 10 , Domaine Universitaire F-38406 St. Martin d'Heres

France

H. POVEL

Institut fir Astronomie

ETH-Zentrum

CH-8092 Zürich

Schweiz

R. RACINE

Canada-France-Hawaii Telescope

Corporation

P.O. Box 1597

Kamuela, HI 96743

U.S.A.

H. RAFF

Carl Zeiss

Postfach $1369 / 9380$

D-7082 Oberkochen

B.R.D.

\section{G. RAFFI}

Buropean Southern Observatory

Karl-Schwarzschild-Str. 2

D-8046 Garching bei Müchen

B. D. D.

\section{P. RAFFIN}

IRAM

Voie 10 - Domaine Universitaire

F-38406 St. Martin d'Heres Cedex France

\section{RAVAUT}

INAG

77, Av. Denfert-Rochereau

F-75014 Paris

France

\section{R. REISS}

European Southern Observatory

Kar1-Schwarzschild-Str. ?

D-8046 Garching bei Minchen

B.R.D.

\section{E.H. RICHARDSON}

Dominion Astrophysical Observatory 5071 W. Saanich Rosd

Victorie, B. C.

V8X 4M6 CANADA

A. RICHAUD

Observatoire de Haute Provence

F-04870 St. Michel I'Observatoire France

\section{0.-G. RICHTER}

European Southern Observatory

Karl-Schwarzschild-Str. 2

D-8046 Garching bei München

B.R.D.

K. RIEGEL

National Science Foundation

1800 G Street N.W.

Washington, D.C. 20550

U.S.A.
A. ROBIN

Observatoire de Besançon

41 bis avenue de I'Observatoire

F-25000 Besançon

France

F. RODDIER

18 Avenue Miltat

F-06100 Nice

France

D. ROUAN

LAIR Observatoire de Meudon

F-92195 Meudon

France

M. ROSA

European Southern Observatory

Karl-Schwarzschild-Str. 2

D-8046 Garching bei München

B.R.D.

\section{SARAZIN}

European Southern Observatory

Karl-Schwarzschild-Str. 2

D-8046 Garching bei Muinchen

B.R.D.

\section{J. SCHMIDT}

Carl Zeiss

Abt. für astronomische Instrumente

Fostfach 1369/1380

D-7082 Oberkochen

B.R.D.

Th. SCHMIDT-KALER

Astronomisches Institut

Ruhr-Universität Bochum

Postfach $1021 \quad 48$

D-4630 BOCHUM 1

B.R.D.

T. SCHMITZ

KRUPP Industrietechnik GmbH

Antenna Department

Franz-Schubert-Str. 1-3

D-4100 Duisburg 14

B.P.D.

\section{SCHNEERMANN}

European Southern Observatory

Karl-Schwerzschlld-Str. 2

D-8046 Garching bei Miunchen

B.R.D.

R. SCHOEMBS

Universitäts-Sternwarte Minchen

Scheinerstrasse 1

D-8000 Minchen 80

B.R.D.

R. SCHULTE-IADBECK

Landessternwarte, Königstuh I

D-6900 Heidelbers 1

B.R.D.

G. SEDMAK

Osservatorio Astronomico di Trieste

Via Tiepolo 11

I-34131 Trieste

Italy

G. SETTI

European Southern Observatory

Karl-Schwarzschild-Str. 2

D-8046 Garching bei München

B.P.D.

P.A. SHAVER

European Southern Observatory

Karl-Schwerzachild-Str. 2

D-8046 Garching bei München

B.R.D. 
K. -H. SIMON

CARL ZEISS

Postfach $1369 / 1380$

D-7082 Oberkochen

B.R.D.

\section{H. SLINGERLAND}

Kapteyn Sterrewacht

R1jksuniversiteit te Groningen

Mensingheweg 20

NL-9301 RODEN

The Netherlands

H.J. SMITH

McDonald Observatory

Astronomy Department, RLM Bldg.

University of Texas

Austin, TX 78712

U.S.A.

H. STANNA

Contraves AG

Schaffhauserstr. 580

$\mathrm{CH}-8052$ Zirich

Schweiz

J.O. STENFIO

Institut fur Astronomie

ETH-Zentrum

$\mathrm{CH}-8092$ Zürich

Schweiz

\section{F. STRAFELIA}

Universitè di Lecce

Dipartimento di Fisica

Via Arnesano

I-73100 Lecce

Italy

D. STRZODA

KRUPP Industrietechnik

Antenna Department

Franz-Schubert-Str. 1-3

D-4100 Dulsburg 14

B.R.D.

\section{J. SURDEJ}

European Southern Observatory

Kerl-Schwarzschild-Str. 2

D-8046 Garching bei Muinchen

B.R.D.

\section{J.P. SWINGS}

IAP Institut d'Astrophysique

Université de Liêge

Av. de Cointe 5

B-4200 Cointe-Ougrée

Belgium

\section{S.c. TAPDE}

Indian Institute of Astrophysics

Koramangale

Bangalore 560034

India

M. TARENGHI

European Southern Observatory

Karl-Schwarzach1ld-Str. 2

D-8046 Garching be1 Minchen

B.R.D.

J. TINBERGEN

Kapteyn Sterrewacht

der Rijksuniversiteit te Groningen

Mensingheweg 20

NL-930† RODEN

The Netherlands

R. TOMELLERI

$A$ D S

Sistemi Avanzati

Vis Cato Plinio 11

I-22030 LIPOMO (COMO)

I taly
K. TOMITA

Tokyo As tronomical Observatory

2-21-1, Osawa, Mi take

Tokyo 181

JAPAN

M.K. TSVETKOV

Dept. of Astronomy and

Nationel Astronomical Observatory

72 Lenin Blvd.

BG-1184 Sofia

Bulgaria

R.G. TULI

University of Texas

Department of Astronomy

RLM 15.308

Austin, 787871

U.S.A.

B.I. ULICH

Steward Observatory

University of Arizone

Tucson, Arizona 85721

U.S.A.

\section{E.A. VALENTIJN}

European Southern Observatory

Karl-Schwarzschild-Str.

D-8046 Garching bei Muinchen

B.R.D.

H. VAN DER LAAN

Sterrewacht

University of Leiden

P.0. Box 9513

NL-2300 RA Leiden

THE NETHERLANDS

\section{M.-P. VERON}

European Southern Observatory

Karl-Schwarzschild-Str. 2

D-8046 Garching bei Muinchen

B.R.D.

\section{P. VERON}

European Southern Observatory

Karl-Schwarzachild-Str. 2

D-8046 Garching bei Miunchen

B.R.D.

\section{G. VIEUX}

Société d'Exploitation Sud Optique

La Boursidière - RN 186

F-92357 Le Plessis Robinson Cedex

France

\section{WAELKENS}

Astronomisch Instituut

Naamses traat 61

B-3000 Leuven

Belgium

D.D. WALKER

University College London

Dept. of Physics \& Astronomy

Gower Street

Lond on WC1E 6BT

U.K.

E.J. WAMPLER

Lick Observatory

University of California

Santa Cruz, CA 95064

U.S.A.

T. WATANABE

Institut fir Theoretische Physik

und Sternwarte der Universität Kie

Physik-Zentrum/Haus Nr. 61 c

Olshausenstrasse

D-2300 KIEL

B.R.D.
M. D. WATERWORTH

University of Tasmania

Box 252 C, G.P.O.

Hobart, Tasmania

AUSTRALIA, 7001

\section{D.W. WEEDMAN}

Pennsylvania State University

Astronomy Department

525 Devey Laboratory

University Park, PA 16802

U.S.A.

G. WEIGELT

Physikalisches Institut

Erwin-Rommel-Str. 1

D-8520 Erlangen

B.R.D.

R. WEST

European Southern Observatory

Karl-Schwarzachild-Str. 2

D-8046 Garching bei München

B.R.D.

\section{J.T. WILLIAMS}

Multiple Mirror Telescope

Smithsonian Astrophysical Observatory

Amado, AZ 85645-0097

U.S.A.

R.V. WILISTROP

Institute of Astronomy

Madingley Road

Cambridge, $\mathrm{CB} 3 \mathrm{OHA}$

U.K.

R. WILSON

European Southern Observatory

Kar1-Schwarz schild-Str. 2

D-8046 Garching bei München

B.R.D.

K. WIRENSTRAND

European Southern Observatory

Karl-Schwarzschild-Str. 2

D-8046 Garching be1 Minchen

B.R.D.

G. WLERICK

Observatoire de Paris

61, Avenue de 1 'Observatoire

F-75014 Paris

France

H. WOHL

Kiepenheuer-Institut fir Sonnenphysik

Schöneckstrasse 6

D-7800 Freibur

B.R.D.

B. WOLF

Landess terawarte, KönigstuhI

D-6900 Heidelberg

B.R.D.

R. WOLF

Max-Planck-Institut fír Astronomie

Königstuhl

D-6900 Heidelberg 1

B.R.D.

L. WOLTJER

European Southern Observatory

Karl-Schwarzschild-Str. 2

D-8046 Garching bef München

B. R.D.

W.-Y. WONG

Dept. of Astronomy

University of Texas

Austin, TX 78712

U.S.A. 
N.J. WOOLF

Steward Observatory

University of Arizona

Tucson, AZ 85721

U.S.A.
J. ZICHA

Institute of Astronomy CSAV

25165 Ondrè jov 251

Czechoslovakia
M. ZIEBELL

European Southern Observatory

Karl-Schwarzschild-Str. 2

D-3046 Garching bel München

B.R.D. 